\title{
Solving the Fuzzy BiLevel Linear Programming with Multiple Followers through Structured Element Method
}

\author{
Shengyue Deng, ${ }^{1}$ Liqian Zhou, ${ }^{2}$ and Xinfan Wang' \\ ${ }^{1}$ School of Science, Hunan University of Technology, Zhuzhou, Hunan 412007, China \\ ${ }^{2}$ School of Computer and Communication, Hunan University of Technology, Zhuzhou, Hunan 412007, China \\ Correspondence should be addressed to Liqian Zhou; zhoulq11@163.com
}

Received 10 May 2014; Accepted 11 June 2014; Published 29 June 2014

Academic Editor: Housheng Su

Copyright (c) 2014 Shengyue Deng et al. This is an open access article distributed under the Creative Commons Attribution License, which permits unrestricted use, distribution, and reproduction in any medium, provided the original work is properly cited.

\begin{abstract}
The optimal solution of fuzzy bilevel linear programming with multiple followers (MFFBLP) model is shown to be equivalent to the optimal solution of the bilevel linear programming with multiple followers by using fuzzy structured element theory. The optimal solution to this model is found out by adopting the Kuhn-Tucker approach. Finally, an illustrative numerical example for this model is also provided to demonstrate the feasibility and efficiency of the proposed method.
\end{abstract}

\section{Introduction}

Bilevel programming introduced by Stackelberg in 1952 [1] has been developed to solve the decentralized planning problem in which decision makers are often arranged within a hierarchical administrative structure. A bilevel programming problem occurs when two decision makers are located at different hierarchical levels. In general, a decision maker at the upper level is termed as the leader and the lower level is termed as the follower $[2,3]$. In the context of bilevel programming, the leader first specifies a strategy; the follower then specifies a strategy so as to optimize the objective with full knowledge of the action of the leader.

Many researches on bilevel programming so far have centered on the linear version of the problem [2-7]. Also two fundamental issues in theory and practice of both bilevel programming problems are mostly concerned: one is how to model a real world bilevel programming and the other is how to find properties and an optimal solution to the bilevel programming problem. There are many such hierarchical optimization problems in the fields of industry, agriculture, finance transportation, and so on [8-11]. But in many practical hierarchical decision making systems, the coefficients of objective functions and constraints sometimes cannot be described by precise values. Hence, it is necessary for us to formulate the decentralized decision making problem with uncertainty as fuzzy models.
At present, the linear bilevel programming in which the coefficients are characterized by fuzzy numbers is called fuzzy linear bilevel programming [12, 13]. Sakawa et al. [4, 5, 14-17] formulated cooperative fuzzy bilevel programming problems and proposed an interactive fuzzy programming approach to solve the problems. From this approach, the concept of a bilevel programming was introduced based on fuzzy number $\lambda$-level sets. At the same time, some researches applied fuzzy set technique to deal with bilevel programming problems. Shih and Stanely Lee [18] applied fuzzy set theory to overcome the computational difficulties in solving bilevel problems. Sinha [19] started from the fuzzy mathematical programming approach to obtain the solution of multilevel linear programming problems. Recently, Zhang et al. [13, 20-22] studied fuzzy bilevel programming problem, which focuses on the situation where the leader or the follower has multiple objectives with fuzzy parameters and all followers share their decision variables, and provided related algorithms based on the $y$ function in fuzzy set theory. Moreover, they have first solved the fuzzy linear bilevel programming problems with a specialized form of membership functions, triangular form, in the fuzzy parameters $[6,12]$. Nevertheless so far the fuzzy linear bilevel programming problem remains the focus of fuzzy multilevel programming problem.

This paper discusses the fuzzy bilevel linear programming with multiple followers (MFFBLP) model. Based on the homeomorphism properties between the bounded real fuzzy 
number and the monotone functions on $[-1,1]$, the comparison of a fuzzy number is changed into a new comparison of monotone function by the definition of fuzzy numbers structured element weighted order. Then the optimal solutions of new derived model is proved equivalent to the optimal solution of the MFFBLP model. The feasibility of the proposed approach is proved by giving a numerical example.

The following of this paper is arranged as follows. In Section 2, some concepts and properties of the fuzzy numbers structured element weighted order are introduced. In Section 3, we study the optimal solution of the MFFBLP model. In Section 4, one numerical example is shown for illustrating the proposed models and approach. Finally, we give some conclusions in Section 5.

\section{Preliminaries}

In this section, some necessary backgrounds and notions of fuzzy structured element theory are presented.

Definition 1 (see [23]). Let $E$ be a fuzzy set on $R$ and $E(x)$ the membership function of $E$. Then, $E$ is called a fuzzy structured element, if (i) $E(0)=1$; (ii) $E(x)$ is a function of monotonous increasing and right continuous on $[-1,0]$ and monotone decreasing and left continuous on $(0,1]$; (iii) $E(x)=0(-\infty<x<-1$ or $1<x<+\infty)$.

Definition 2 (see [23]). $E$ is called a canonical fuzzy structured element, if (i) $\forall x \in(-1,1), E(x)>0$; (ii) $E(x)$ is continuous and strictly monotone increasing on $[-1,0]$ and strictly monotone decreasing and continuous on $(0,1]$.

Definition 3 (see [23]). $E$ is called a symmetrical fuzzy structured element, if $E(-x)=E(x)$.

Lemma 4 (see [23]). Let $E$ be a fuzzy structured element and $E(x)$ is its membership function; the function $f(x)$ is continuous and monotone on $[-1,1]$; then $f(E)$ is a fuzzy number, and the membership function of $f(E)$ is $E\left(f^{-1}(x)\right)$, where $f^{-1}(x)$ is rotational symmetry function for variables $x$ and $y$, if $f$ is a strictly monotone function, then $f^{-1}(x)$ is the inverse function of $f(x)$.

Lemma 5 (see [24]). For a given canonical fuzzy structured element $E$ and any finite fuzzy number $\widetilde{A}$, there always exists a monotone bounded function $f$ on $[-1,1]$, having the form $\widetilde{A}=f(E)$.

Lemma 6 (see [25]). Let the triangular fuzzy number $\widetilde{A}=$ $(a, b, c), E$ is a fuzzy structured element, and its membership function is

$$
E(x)= \begin{cases}1+x, & -1 \leq x \leq 0 \\ 1-x, & 0 \leq x \leq 1 \\ 0, & \text { others }\end{cases}
$$

Then arbitrary bounded triangular fuzzy number can be generated by $E$ and the monotone bounded function is

$$
f(x)= \begin{cases}(b-a) x+b, & -1 \leq x \leq 0 \\ (c-b) x+b, & 0 \leq x \leq 1 \\ 0, & \text { others }\end{cases}
$$

Therefore, we easily get the conclusion $\widetilde{A}=f(E)$.

Remark 7. The class of all bounded fuzzy numbers is denoted by $\widetilde{N}_{C}(R)$, and let $E$ be a canonical fuzzy structured element.

Definition 8 (see [23]). Suppose $\widetilde{A}_{1}, \widetilde{A}_{2} \in \widetilde{N}_{C}(R)$. Its structured element representation is $\widetilde{A}_{i}=f_{i}(E), i=1,2$, respectively, where $E$ is given a canonical fuzzy structured element and its membership function is $E(x), f_{1}(x)$ and $f_{2}(x)$ are the same sequence monotonic functions on $[-1,1]$, respectively. By the following formula

$$
\begin{aligned}
\widetilde{A}_{1} & \leq \widetilde{A}_{2} \Longleftrightarrow F\left(\widetilde{A}_{1}, \widetilde{A}_{2}\right) \\
& =\int_{-1}^{1} E(x)\left(f_{1}(x)-f_{2}(x)\right) d x \\
& =\int_{-1}^{1} E(x) f_{1}(x) d x-\int_{-1}^{1} E(x) f_{2}(x) d x \leq 0
\end{aligned}
$$

to determine the binary relation " $\leq$ " is a total order on $\widetilde{N}_{C}(R)$ and then called fuzzy numbers structured element weighted order.

Lemma 9 (see [23]). Let $E$ be a symmetrical fuzzy structured element, $f_{1}(x)$ and $f_{2}(x)$ are the same sequence monotonic functions on $[-1,1]$, the fuzzy number $\widetilde{A}_{1}=f_{1}(E)$, and $\widetilde{A}_{2}=$ $f_{2}(E)$, then

$$
\begin{aligned}
\widetilde{A}_{1}+\widetilde{A}_{2} & =f_{1}(E)+f_{2}(E), \\
\widetilde{A}_{1}-\widetilde{A}_{2} & =f_{1}(E)+f_{2}^{\tau}(E), \\
k \widetilde{A}_{1} & =|k| f_{1}^{\tau}(E), \\
k \widetilde{A}_{2} & =|k| f_{2}^{\tau}(E),
\end{aligned}
$$

when $k \geq 0, f_{1}^{\tau}(E)=f_{1}(E)$, and $f_{2}^{\tau}(E)=f_{2}(E)$; when $k<0$, $f_{1}^{\tau}(E)=-f_{1}(-E)$, and $f_{2}^{\tau}(E)=-f_{2}(-E)$. 25]. 


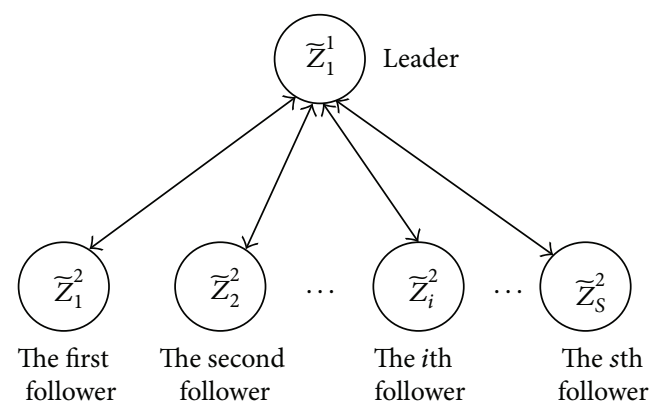

FIGURE 1: Fuzzy bilevel linear programming with multiple followers.

\section{Fuzzy Bilevel Linear Programming with Multiple Followers}

Figure 1 shows the fuzzy bilevel linear programming with multiple followers model (MFFBLP), and this mathematical model is defined as follows:

$$
\begin{aligned}
& \min _{x_{i}} \widetilde{Z}_{1}^{1}=\sum_{i=1}^{N} \widetilde{c}_{i}^{1} x_{i}+\sum_{s=1}^{S} \sum_{j=1}^{M} \widetilde{d}_{s j}^{1} y_{s j} ; \\
& \text { s.t. } \quad \text { where } y_{s j}(s=1,2, \ldots, S ; j=1,2, \ldots, M)
\end{aligned}
$$

is the solution of the lower level problem:

$$
\begin{aligned}
& \min _{y_{s j}} \widetilde{Z}_{s}^{2}=\sum_{i=1}^{N} \widetilde{c}_{s i}^{2} x_{i}+\sum_{j=1}^{M} \widetilde{d}_{s j}^{2} y_{s j} \\
& \text { s.t } \quad \sum_{i=1}^{N} \widetilde{a}_{t i}^{s} x_{i}+\sum_{j=1}^{M} \widetilde{b}_{t j}^{s} y_{s j} \leq \widetilde{e}_{t}^{s} ; \\
& \qquad x_{i} \geq 0 ; y_{s j} \geq 0 ; \\
& i=1,2, \ldots, N ; \quad j=1,2, \ldots, M ; \quad s=1,2, \ldots, S ;
\end{aligned}
$$

where $\widetilde{Z}_{1}^{1}$ is the leader's objective function and $\widetilde{Z}_{s}^{2}$ is the sth follower's objective function; $x_{i}, y_{s j} \in$ $R ; \widetilde{c}_{i}^{1}, \widetilde{c}_{s i}^{2}, \widetilde{d}_{s j}^{1}, \widetilde{d}_{s j}^{2}, \widetilde{a}_{t i}^{s}, \widetilde{b}_{t j}^{s}, \widetilde{e}_{t}^{s} \in \widetilde{N}_{C}(R)$.

Theorem 10. Suppose that $\widetilde{Z}_{1}^{1}=G_{1}^{1}(E), \widetilde{Z}_{s}^{2}=G_{s}^{2}(E), \widetilde{c}_{i}^{1}=$ $f_{i}^{1}(E), \widetilde{c}_{s i}^{2}=f_{s i}^{2}(E), \widetilde{d}_{s j}^{1}=F_{s j}^{1}(E), \widetilde{d}_{s j}^{2}=F_{s j}^{2}(E), \widetilde{a}_{t i}^{s}=\varphi_{t i}^{s}(E)$, $\tilde{b}_{t j}^{s}=g_{t j}^{s}(E), \widetilde{e}_{t}^{s}=\psi_{t}^{s}(E)$, and $M_{1}^{1}=\int_{-1}^{1} E(t) G_{1}^{1}(t) d t$, if $E$ is a canonical fuzzy structured element, $G_{1}^{1}(t), G_{s}^{2}(t), f_{i}^{1}(t)$, $f_{s i}^{2}(t), F_{s j}^{1}(t), F_{s j}^{2}(t), \varphi_{t i}^{s}(t), g_{t j}^{s}(t)$, and $\psi_{t}^{s}(t)$ are monotonous increasing functions, then the model (5) is equivalent to the following model:

$\min _{x_{i}} M_{1}=\sum_{i=1}^{N} x_{i} \int_{-1}^{1} E(t) f_{i}^{1}(t) d t+\sum_{s=1}^{S} \sum_{j=1}^{M} y_{s j} \int_{-1}^{1} E(t) F_{s j}^{1}(t) d t$

s.t. where $y_{s j}(s=1,2, \ldots, S ; j=1,2, \ldots, M)$ is the

solution of the lower level problem:

$\min _{y_{s j}} M_{s}^{2}=\sum_{i=1}^{N} x_{i} \int_{-1}^{1} E(t) f_{s i}^{2}(t) d t+\sum_{j=1}^{M} y_{s j} \int_{-1}^{1} E(t) F_{s j}^{2}(t) d t$

s.t $\quad \sum_{i=1}^{N} x_{i} \int_{-1}^{1} E(t) \varphi_{t i}^{s}(t) d t+\sum_{j=1}^{M} y_{s j} \int_{-1}^{1} E(t) g_{t j}^{s}(t) d t$

$$
\begin{aligned}
& \leq \int_{-1}^{1} E(t) \psi_{t}^{s}(t) d t ; \\
& \quad x_{i} \geq 0 ; y_{s j} \geq 0 ; \\
& i=1,2, \ldots, N ; \quad j=1,2, \ldots, M ; \quad s=1,2, \ldots, S ; \\
& t=1,2, \ldots, T .
\end{aligned}
$$

Proof. By Definition 8, we know that comparing the size of fuzzy number $\widetilde{Z}_{1}^{1}$ by $M_{1}^{1}=\int_{-1}^{-1} E(t) G_{1}^{1}(t) d t$ in model (5), then

$$
\begin{aligned}
\widetilde{Z}_{1}^{1} & =G_{1}^{1}(E)=\sum_{i=1}^{N} \widetilde{c}_{i}^{1} x_{i}+\sum_{s=1}^{S} \sum_{j=1}^{M} \widetilde{d}_{s j}^{1} y_{s j} \\
& =\sum_{i=1}^{N} f_{i}^{1}(E) x_{i}+\sum_{s=1}^{S} \sum_{j=1}^{M} F_{s j}^{1}(E) y_{s j} ;
\end{aligned}
$$

because $f_{i}^{1}(t)$ and $F_{s j}^{1}(t)$ are monotonous increasing functions; then

$$
\begin{aligned}
M_{1}^{1} & =\int_{-1}^{1} E(t) G_{1}^{1}(t) d t \\
& =\int_{-1}^{1} E(t)\left[\sum_{i=1}^{N} f_{i}^{1}(t) x_{i}+\sum_{s=1}^{S} \sum_{j=1}^{M} F_{s j}^{1}(t) y_{s j}\right] d t \\
& =\int_{-1}^{1} E(t) \sum_{i=1}^{N} f_{i}^{1}(t) x_{i} d t+\int_{-1}^{1} E(t) \sum_{s=1}^{S} \sum_{j=1}^{M} F_{s j}^{1}(t) y_{s j} d t \\
& =\sum_{i=1}^{N} x_{i} \int_{-1}^{1} E(t) f_{i}^{1}(t) d t+\sum_{s=1}^{S} \sum_{j=1}^{M} y_{s j} \int_{-1}^{1} E(t) F_{s j}^{1}(t) d t .
\end{aligned}
$$

In the same way, we get

$$
\begin{aligned}
M_{s}^{2} & =\int_{-1}^{1} E(t) G_{s}^{2}(t) d t \\
& =\sum_{i=1}^{N} x_{i} \int_{-1}^{1} E(t) f_{s i}^{2}(t) d t+\sum_{j=1}^{M} y_{s j} \int_{-1}^{1} E(t) F_{s j}^{2}(t) d t .
\end{aligned}
$$


By Lemmas 6 and 9, we obtain that

$$
\begin{aligned}
& \sum_{i=1}^{N} x_{i} \int_{-1}^{1} E(t) \varphi_{t i}^{s}(t) d t+\sum_{j=1}^{M} y_{s j} \int_{-1}^{1} E(t) g_{t j}^{s}(t) d t \\
& \quad \leq \int_{-1}^{1} E(t) \psi_{t}^{s}(t) d t .
\end{aligned}
$$

The proof is completed.

\section{Algorithm and Numerical Example}

4.1. Algorithm. By using Theorem 10, we give all steps of the approach for the proposed MFFBLP model.

Step 1. If the fuzzy number is triangular fuzzy number, according to Lemma 6 and $E$ expression, we have $\mu_{\widetilde{A}}(t)=$ $E\left(f^{-1}(t)\right)$ and then get $f_{i}^{1}(t), f_{s i}^{2}(t), F_{s j}^{1}(t), F_{s j}^{2}(t), \varphi_{t i}^{s}(t), g_{t j}^{s}(t)$, and $\psi_{t}^{s}(t)$.

Step 2. Computation formula is as follows: $\int_{-1}^{1} E(t) f_{i}^{1}(t) d t$, $\int_{-1}^{1} E(t) f_{s i}^{2}(t) d t, \quad \int_{-1}^{1} E(t) F_{s j}^{1}(t) d t, \quad \int_{-1}^{1} E(t) F_{s j}^{2}(t) d t, \quad \int_{-1}^{1} E(t)$ $\varphi_{t i}^{s}(t) d t, \int_{-1}^{1} E(t) g_{t j}^{s}(t) d t$, and $\int_{-1}^{1} E(t) \psi_{t}^{s}(t) d t$, and plug in model (5).

Step 3. According to Theorem 10, the MFFBLP model is transformed into the classical bilevel linear programming with multiple followers model whose optimal solution of model (6) can be derived from Kuhn-Tucker's approach [7].

Step 4. After the optimal solution of model (6) is plugged into model (5), we get the optimal solution of the MFFBLP model.

4.2. Numerical Example. Consider the following MFFBLP problem with $x_{1} \in R^{1}, x_{2} \in R^{1}, y_{11} \in R^{1}$, and $y_{21} \in R^{1}$. Consider

$\min _{x_{1}, x_{2}} \widetilde{Z}_{1}^{1}\left(x_{1}, x_{2}, y_{11}, y_{21}\right)=\widetilde{3.1} x_{1}+\widetilde{8} x_{2}+\widetilde{6.9} y_{11}+\widetilde{11} y_{21}$

s.t. where given $x_{1}, x_{2}$, and $y_{11}, y_{21}$ solve the following problem.

$\min _{y_{11}} \widetilde{Z}_{1}^{2}\left(x_{1}, x_{2}, y_{11}\right)=\widetilde{2} x_{1}+\widetilde{1} x_{2}-\widetilde{1} y_{11}$

s.t. $\tilde{6} x_{1}-\widetilde{1} x_{2}+\widetilde{13} y_{11} \leq \widetilde{15}$,

$\widetilde{5} x_{1}+\widetilde{6.9} y_{11} \leq \widetilde{15}$

$-\widetilde{4} x_{2}+\widetilde{25} y_{11} \leq \widetilde{3.1}$

$$
\begin{array}{ll}
\min _{y_{21}} & \widetilde{Z}_{2}^{2}\left(x_{1}, x_{2}, y_{21}\right)=\widetilde{15} x_{1}-\widetilde{1} x_{2}+\widetilde{80} y_{21} ; \\
\text { s.t. } \quad & \widetilde{1} x_{1}+\widetilde{1} x_{2}-\widetilde{6.9} y_{21} \leq \widetilde{10}, \\
& \widetilde{40} x_{1}+\widetilde{1} y_{21} \leq \widetilde{5} \\
& x_{1} \geq 0, \quad x_{2} \geq 0, \quad y_{11} \geq 0, \quad y_{21} \geq 0,
\end{array}
$$

where the triangular fuzzy numbers $\widetilde{1}=(0,1,2), \widetilde{2}=$ $(1.5,2,2.5), \widetilde{3.1}=(2,3.1,3.6), \widetilde{4}=(2,4,6), \widetilde{5}=(4.7,5,5.3)$, $\widetilde{6}=(5,6,7), \widetilde{6.9}=(6.5,6.9,7.9), \widetilde{8}=(7.5,8,8.5), \widetilde{10}=$ $(8,10,12), \widetilde{11}=(10.7,11,11.3), \widetilde{13}=(12,13,14), \widetilde{15}=$ $(14.5,15,15.5), \widetilde{25}=(23,25,27), \widetilde{40}=(39,40,41)$, and $\widetilde{80}=$ $(77,80,83)$.

Step 1. By Lemma 6, we have

$$
\begin{aligned}
& f_{1}^{1}(t)=\psi_{3}^{1}(t)= \begin{cases}1.1 t+3.1, & -1 \leq t \leq 0 \\
0.5 t+3.1, & 0 \leq t \leq 1 \\
0, & \text { others }\end{cases} \\
& f_{2}^{1}(t)= \begin{cases}0.5 t+8, & -1 \leq t \leq 0 \\
0.5 t+8, & 0 \leq t \leq 1 \\
0, & \text { others, }\end{cases} \\
& F_{11}^{1}(t)=g_{21}^{1}(t)= \begin{cases}0.4 t+6.9, & -1 \leq t \leq 0 \\
t+6.9, & 0 \leq t \leq 1 \\
0, & \text { others }\end{cases} \\
& F_{21}^{1}(t)= \begin{cases}0.3 t+11, & -1 \leq t \leq 0 \\
0.3 t+11, & 0 \leq t \leq 1 \\
0, & \text { others }\end{cases} \\
& f_{11}^{2}(t)= \begin{cases}0.5 t+2, & -1 \leq t \leq 0 \\
0.5 t+2, & 0 \leq t \leq 1 \\
0, & \text { others }\end{cases} \\
& f_{12}^{2}(t)=\varphi_{11}^{2}(t)=\varphi_{12}^{2}(t)=g_{21}^{2}(t)= \begin{cases}t+1, & -1 \leq t \leq 0 \\
t+1, & 0 \leq t \leq 1 \\
0, & \text { others }\end{cases} \\
& F_{11}^{2}(t)=\varphi_{12}^{1}(t)=f_{22}^{2}(t)= \begin{cases}t-1, & -1 \leq t \leq 0 \\
t-1, & 0 \leq t \leq 1 \\
0, & \text { others }\end{cases} \\
& \varphi_{11}^{1}(t)= \begin{cases}t+6, & -1 \leq t \leq 0 \\
t+6, & 0 \leq t \leq 1 \\
0, & \text { others, }\end{cases} \\
& g_{11}^{1}(t)= \begin{cases}t+13, & -1 \leq t \leq 0 \\
t+13, & 0 \leq t \leq 1 \\
0, & \text { others, }\end{cases}
\end{aligned}
$$




$$
\begin{aligned}
& \psi_{1}^{1}(t)=\psi_{2}^{1}(t)=f_{21}^{2}(t)= \begin{cases}0.5 t+15, & -1 \leq t \leq 0 \\
0.5 t+15, & 0 \leq t \leq 1 \\
0, & \text { others }\end{cases} \\
& \varphi_{21}^{1}(t)=\psi_{1}^{2}(t)= \begin{cases}0.3 t+5, & -1 \leq t \leq 0 \\
0.3 t+5, & 0 \leq t \leq 1 \\
0, & \text { others }\end{cases} \\
& \varphi_{32}^{1}(t)= \begin{cases}2 t-4, & -1 \leq t \leq 0 \\
2 t-4, & 0 \leq t \leq 1 \\
0, & \text { others }\end{cases} \\
& g_{31}^{1}(t)= \begin{cases}2 t+25, & -1 \leq t \leq 0 \\
2 t+25, & 0 \leq t \leq 1 \\
0, & \text { others }\end{cases} \\
& F_{21}^{2}(t)= \begin{cases}3 t+80, & -1 \leq t \leq 0 \\
3 t+80, & 0 \leq t \leq 1 \\
0, & \text { others }\end{cases} \\
& g_{11}^{2}(t)= \begin{cases}t-6.9, & -1 \leq t \leq 0 \\
0.4 t-6.9, & 0 \leq t \leq 1 \\
0, & \text { others }\end{cases} \\
& \psi_{1}^{2}(t)= \begin{cases}2 t+10, & -1 \leq t \leq 0 \\
2 t+10, & 0 \leq t \leq 1 \\
0, & \text { others }\end{cases} \\
& \varphi_{11}^{2}(t)= \begin{cases}t+40, & -1 \leq t \leq 0 \\
t+40, & 0 \leq t \leq 1 \\
0, & \text { others. }\end{cases}
\end{aligned}
$$

Step 2. Compute $\int_{-1}^{1} f_{1}^{1}(t) E(t) d t=3, \int_{-1}^{1} f_{2}^{1}(t) E(t) d t=8$, $\int_{-1}^{1} F_{11}^{1}(t) E(t) d t=7, \int_{-1}^{1} F_{21}^{1}(t) E(t) d t=11, \int_{-1}^{1} f_{11}^{2}(t) E(t) d t=$ 2, $\int_{-1}^{1} f_{12}^{1}(t) E(t) d t=1, \int_{-1}^{1} F_{11}^{2}(t) E(t) d t=-1, \int_{-1}^{1} \varphi_{11}^{1}$ $(t) E(t) d t=6, \int_{-1}^{1} g_{11}^{1}(t) E(t) d t=13, \int_{-1}^{1} \psi_{1}^{1}(t) E(t) d t=$ $15, \int_{-1}^{1} \varphi_{21}^{1}(t) E(t) d t=5, \int_{-1}^{1} \varphi_{32}^{1}(t) E(t) d t=-4, \int_{-1}^{1} g_{31}^{1}(t)$ $E(t) d t=25, \int_{-1}^{1} F_{21}^{2}(t) E(t) d t=80, \int_{-1}^{1} g_{11}^{2}(t) E(t) d t=-7$, $\int_{-1}^{1} \psi_{1}^{2}(t) E(t) d t=10$, and $\int_{-1}^{1} \varphi_{11}^{2}(t) E(t) d t=40$.

Step 3. By Theorem 10, the original problem is equivalent to the following bilevel linear programming with multiple followers' problem:

$$
\min _{x_{1}, x_{2}} M_{1}^{1}\left(x_{1}, x_{2}, y_{11}, y_{21}\right)=3 x_{1}+8 x_{2}+7 y_{11}+11 y_{21} ;
$$

s.t. where given $x_{1}, x_{2}$, and $y_{11}, y_{21}$ solve the following problem.

$$
\begin{array}{ll}
\min _{y_{11}} & M_{1}^{2}\left(x_{1}, x_{2}, y_{11}\right)=2 x_{1}+x_{2}-y_{11} ; \\
\text { s.t. } & 6 x_{1}-x_{2}+13 y_{11} \leq 15, \\
& 5 x_{1}+7 y_{11} \leq 15, \\
& -4 x_{2}+25 y_{11} \leq 3 ; \\
& \min _{y_{21}} \quad M_{2}^{2}\left(x_{1}, x_{2}, y_{21}\right)=15 x_{1}-x_{2}+80 y_{21} ; \\
\text { s.t. } \quad & x_{1}+x_{2}-7 y_{21} \leq 10, \\
& 40 x_{1}+y_{21} \leq 5 ; \\
& x_{1} \geq 0, \quad x_{2} \geq 0, \quad y_{11} \geq 0, \quad y_{21} \geq 0 .
\end{array}
$$

Step 4. We use the Kuhn-Tucker approach to get an optimal solution; the solution of the problem is as follows:

$$
\begin{gathered}
\left(x_{1}, x_{2}, y_{11}, y_{21}\right)=(0.1182,11.7878,2.0061,0.2723) \\
M_{1}^{1}=111.6946, \quad M_{1}^{2}=10.0181, \quad M_{2}^{2}=11.7692 \\
\widetilde{Z}_{1}^{1}=\left(\underline{Z}_{1}^{1}, Z_{1}^{1}, \bar{Z}_{1}^{1}\right)=(104.5982,111.5062,119.5470) \\
\widetilde{Z}_{1}^{2}=\left(\underline{Z}_{1}^{2}, Z_{1}^{2}, \bar{Z}_{1}^{2}\right)=(-3.8349,10.0181,23.8711) \\
\widetilde{Z}_{2}^{2}=\left(\underline{Z}_{2}^{2}, Z_{2}^{2}, \bar{Z}_{2}^{2}\right)=(-0.8946,11.7692,24.4330)
\end{gathered}
$$

The example illustrates how to solve a MFFBLP problem and obtains an optimal solution by using the proposed approach.

\section{Conclusions}

A real world bilevel decision problem may be modeled to have fuzzy coefficients. In this paper, we investigated the MFFBLP model and solved this complex problem by using the fuzzy structured element method. Further study includes the development of models and methods for fuzzy multilevel programming. We will also explore effective applications of the proposed techniques.

\section{Conflict of Interests}

The authors declare that there is no conflict of interests regarding the publication of this paper.

\section{Acknowledgments}

The work was supported by the Scientific Research Fund of Hunan Provincial Education Department of China (Grant nos. 14C0350 and 13A004), the NSF of Hunan Province of China (Grant nos. 13JJ3109 and 14JJ3127), and the Humanities and Social Science Foundation of the Ministry of Education of China (Grant no. 12YJA630114). 


\section{References}

[1] H. V. Stackelberg, The Theory of the Market Economy, Oxford University Press, Oxford, UK, 1952.

[2] W. F. Bialas and M. H. Karwan, "Two-level linear programming," Management Science, vol. 30, no. 8, pp. 1004-1020, 1984.

[3] S. Dempe, Foundations of Bilevel Programming, vol. 61 of Nonconvex Optimization and its Applications, Kluwer Academic, Dordrecht, The Netherlands, 2002.

[4] M. Sakawa and I. Nishizaki, "Interactive fuzzy programming for decentralized two-level linear programming problems," Fuzzy Sets and Systems, vol. 125, no. 3, pp. 301-315, 2002.

[5] M. Sakawa, H. Katagiri, and T. Matsui, "Stackelberg solutions for fuzzy random two-level linear programming through probability maximization with possibility," Fuzzy Sets and Systems, vol. 188 , pp. 45-57, 2012.

[6] G. Zhang and J. Lu, "The definition of optimal solution and an extended Kuhn-Tucker approach for fuzzy linear bilevel programming," The IEEE Computational Intelligence Bulletin, vol. 5, pp. 1-7, 2005.

[7] J. Lu, C. Shi, and G. Zhang, "On bilevel multi-follower decision making: general framework and solutions," Information Sciences: An International Journal, vol. 176, no. 11, pp. 1607-1627, 2006.

[8] H. A. Gil, F. D. Galiana, and E. L. da Silva, "Nodal price control: a mechanism for transmission network cost allocation," IEEE Transactions on Power Systems, vol. 21, no. 1, pp. 3-10, 2006.

[9] H. Yang, X. Zhang, and Q. Meng, "Stackelberg games and multiple equilibrium behaviors on networks," Transportation Research Part B: Methodological, vol. 41, no. 8, pp. 841-861, 2007.

[10] K. Kogan and C. S. Tapiero, "Optimal co-investment in supply chain infrastructure," European Journal of Operational Research, vol. 192, no. 1, pp. 265-276, 2009.

[11] Z. Yao, S. C. H. Leung, and K. K. Lai, "Manufacturer's revenuesharing contract and retail competition," European Journal of Operational Research, vol. 186, no. 2, pp. 637-651, 2008.

[12] G. Zhang and J. Lu, "Model and approach of fuzzy bilevel decision making for logistics planning problem," Journal of Enterprise Information Management, vol. 20, no. 2, pp. 178-197, 2007.

[13] Y. Gao, G. Zhang, J. Ma, and J. Lu, "A $\lambda$-cut and goalprogramming-based algorithm for fuzzy-linear multipleobjective bilevel optimization," IEEE Transactions on Fuzzy Systems, vol. 18, no. 1, pp. 1-13, 2010.

[14] M. Sakawa, I. Nishizaki, and Y. Uemura, "Interactive fuzzy programming for multi-level linear programming problems with fuzzy parameters," Fuzzy Sets and Systems, vol. 109, no. 1, pp. 3-19, 2000.

[15] M. Sakawa and K. Yauchi, "Interactive decision making for multiobjective nonconvex programming problems with fuzzy numbers through coevolutionary genetic algorithms," Fuzzy Sets and Systems, vol. 114, no. 1, pp. 151-165, 2000.

[16] M. Sakawa and I. Nishizaki, "Interactive fuzzy programming for two-level linear fractional programming problems," Fuzzy Sets and Systems, vol. 119, no. 1, pp. 31-40, 2001.

[17] M. Sakawa and I. Nishizaki, "Interactive fuzzy programming for two-level nonconvex programming problems with fuzzy parameters through genetic algorithms," Fuzzy Sets and Systems, vol. 127, no. 2, pp. 185-197, 2002.

[18] H. Shih and E. Stanley Lee, "Compensatory fuzzy multiple level decision making," Fuzzy Sets and Systems, vol. 114, no. 1, pp. 7187, 2000.
[19] S. Sinha, "Fuzzy programming approach to multi-level programming problems," Fuzzy Sets and Systems, vol. 136, no. 2, pp. 189-202, 2003.

[20] G. Zhang, J. Lu, and T. Dillon, "Decentralized multi-objective bilevel decision making with fuzzy demands," Knowledge-Based Systems, vol. 20, no. 5, pp. 495-507, 2007.

[21] G. Zhang, J. Lu, and Y. Gao, "Fuzzy bilevel programming: multiobjective and multi-follower with shared variables," International Journal of Uncertainty, Fuzziness and Knowledge-Based Systems, vol. 16, pp. 105-133, 2008.

[22] G. Zhang, J. Lu, and Y. Gao, "An algorithm for fuzzy multiobjective multi-follower partial cooperative bilevel programming," Journal of Intelligent and Fuzzy Systems, vol. 19, no. 4-5, pp. 303-319, 2008.

[23] S. Guo, Principle of Mathematical Analysis Based on Structured Element, Northeast University Press, Shenyang, China, 2004.

[24] S. Z. Guo, "Common representation method for fuzzy-valued functions based on structured elements," Fuzzy Systems and Mathematics, vol. 19, no. 1, pp. 82-86, 2005.

[25] S. Guo, "Comparison and sequencing of fuzzy numbers based on the method of structured element," System Engineering Theory and Practice, vol. 29, no. 3, pp. 106-111, 2009. 


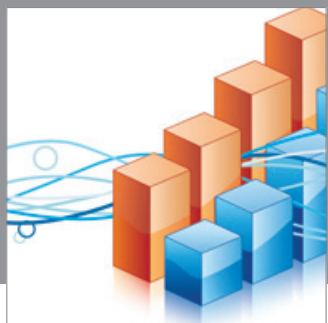

Advances in

Operations Research

mansans

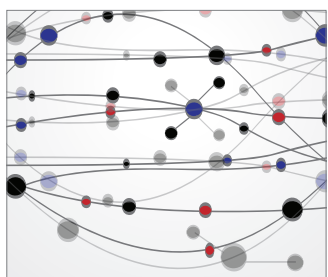

The Scientific World Journal
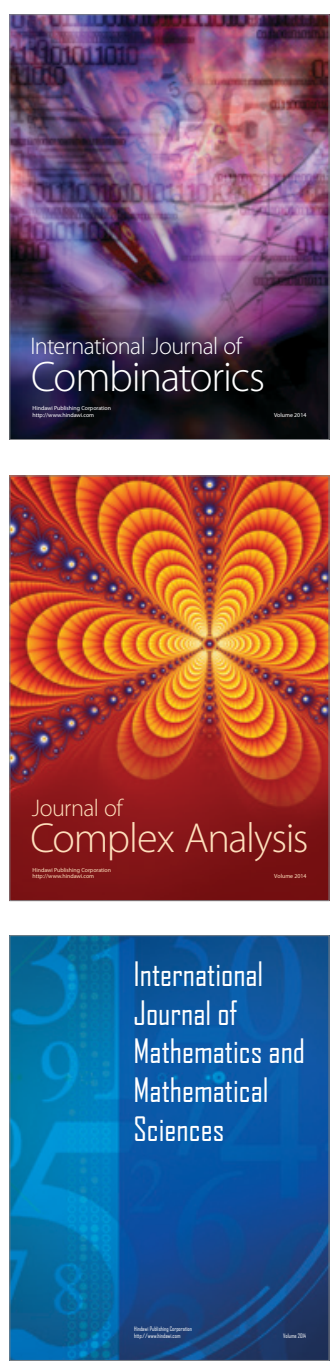
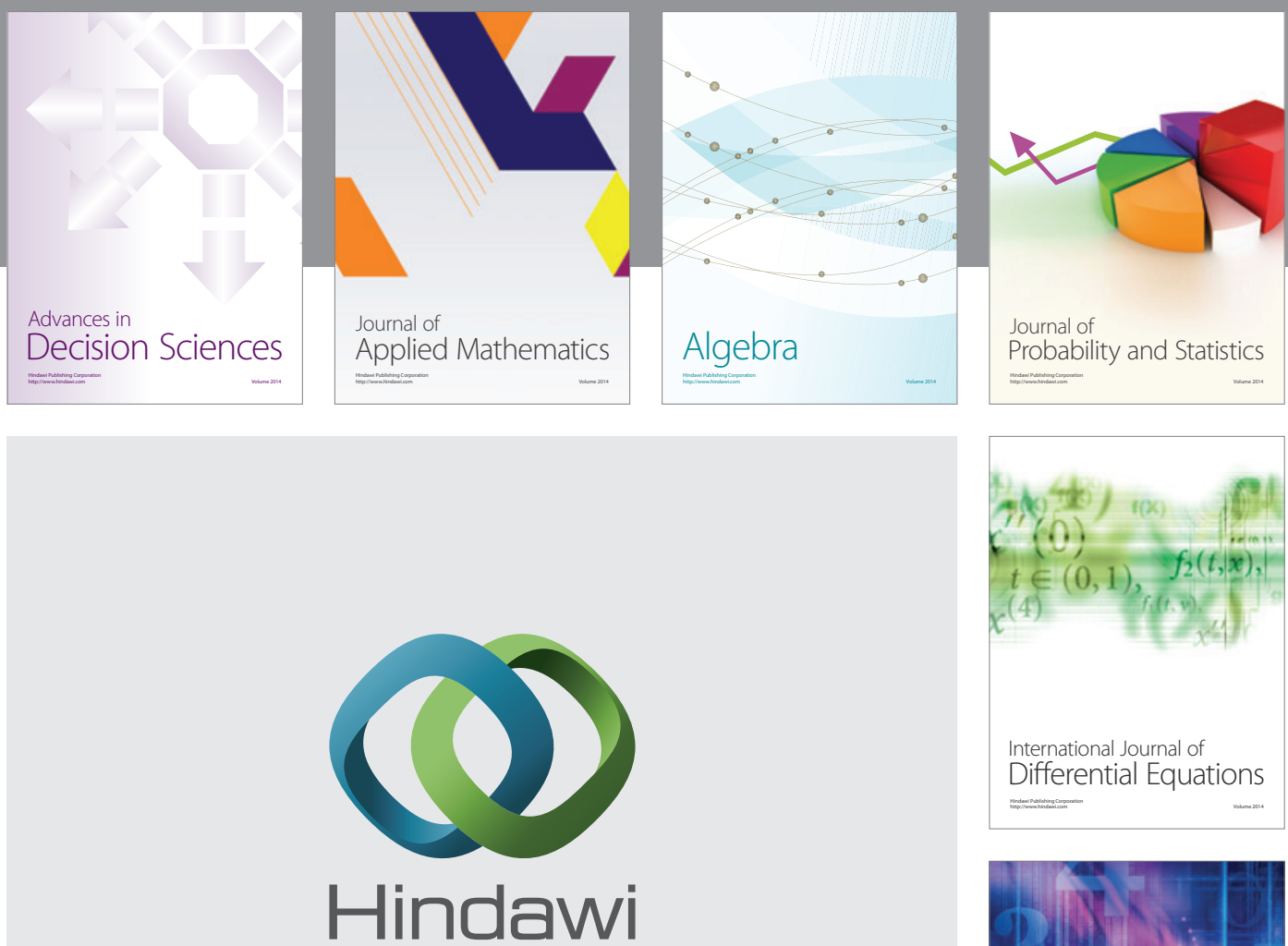

Submit your manuscripts at http://www.hindawi.com
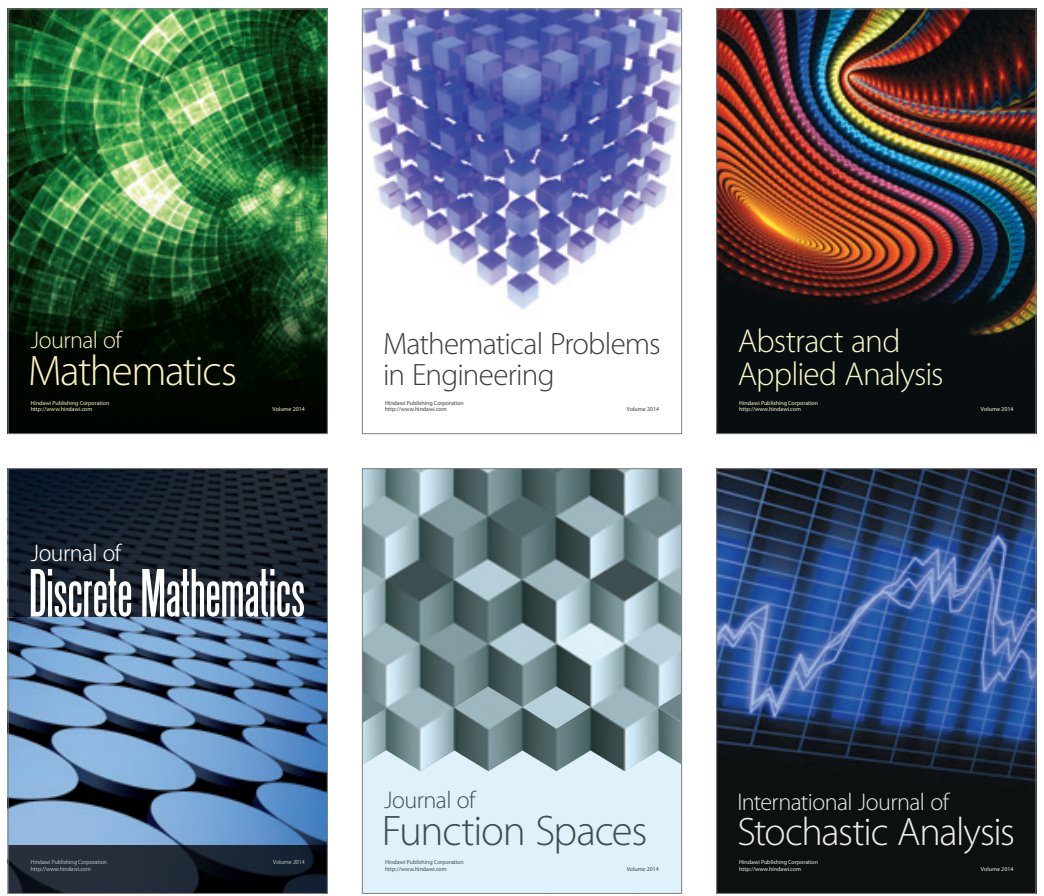

Journal of

Function Spaces

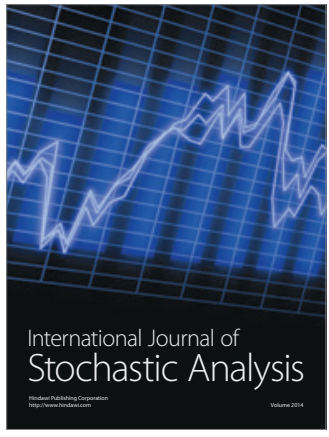

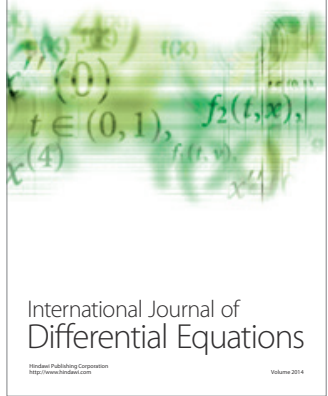
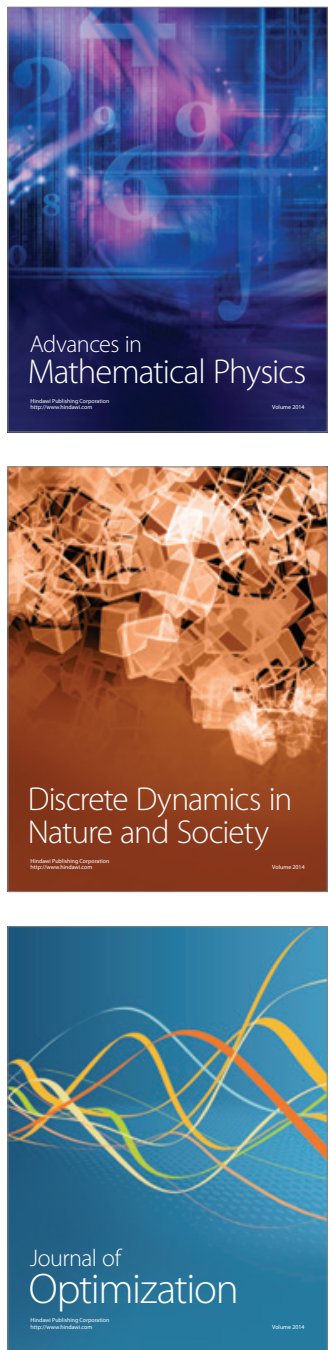